\title{
PROPER MAPPINGS AND THE MINIMUM DIMENSION OF A COMPACTIFICATION OF A SPACE
}

\author{
JAMES KEESLING
}

\begin{abstract}
In this paper it is shown that for each positive integer $n$ there is a locally compact Hausdorff space $X$ having the property that $\operatorname{dim} X=n$ and in addition having the property that if $f(X)=Y$ is a proper mapping, then $\operatorname{dim} Y \geqq n$. Using this result it is shown that there is a space $Y$ having the property that min $\operatorname{dim} Y=n$ with a point $p \in Y$ with $\min \operatorname{dim} Y-\{p\}=0$.
\end{abstract}

Introduction. A proper mapping is a closed continuous function $f: X \rightarrow Y$ such that $f^{-1}(y)$ is compact for all $y \in Y$. In this paper it is shown that for each positive integer $n$ there is a locally compact Hausdorff space $X$ having the property that $\operatorname{dim} X=n$ and in addition has the property that if $f(X)=Y$ is a proper mapping, then $\operatorname{dim} Y \geqq n$. For $n=2$ such a space was shown to exist in [5] in answer to a question posed by Isbell [4, pp. 119-120].

Using this result it is then shown that for each positive integer $n$ there is a space $X$ having the property that the minimum dimension of a compactification for $X$ is $n$ and that there is a point $p \in X$ such that $X-\{p\}$ has a 0 -dimensional compactification. In the terminology of Isbell [4, p. 97], min $\operatorname{dim} X=n$ with $\min \operatorname{dim} X-\{p\}=0$.

Notation. Throughout the paper $X$ and $Y$ denote completely regular Hausdorff spaces. By $\operatorname{dim} X$ is meant the modified Lebesgue covering dimension of $X\left[2\right.$, p. 243] or $\left[4\right.$, p. 97].$^{1}$ By $\min \operatorname{dim} X$ is meant the minimum dimension of a compactification of $X[4, \mathrm{p} .97]$. The cardinals are considered to be a subset of the ordinals in the natural way. The infinite cardinals will be denoted by $\omega_{0}, \omega_{1}, \omega_{2}, \ldots$ where $\omega_{0}$ is the first infinite ordinal, $\omega_{1}$ is the first uncountable ordinal, etc. If $\alpha$ is an ordinal, then by $[0, \alpha)$ will be meant the set of all ordinals less than $\alpha$ except that $[0,1)$ will denote the half-open unit interval. The set $[0, \alpha)$ will be thought of as a topological space with the order topology. By $L(\alpha)$ will be meant the set $[0, \alpha) \times[0,1)$ endowed with the lexicographic order and given the order topology. The space $L(\alpha)$ is called a long line.

Received by the editors December 17, 1970.

AMS 1970 subject classifications. Primary 54F45, 54D35; Secondary 54C10.

Key words and phrases. Dimension, proper mapping, locally compact space, compactification.

1 One could define the modified Lebesgue covering dimension of a space $X$ to be the ordinary Lebesgue covering dimension of $\beta X$.

Copyright @ 1971, American Mathematical Society 
I. Proper mappings. In this section we show that if $X=\prod_{i=1}^{n} L\left(\omega_{i}\right)$ and if $f(X)=Y$ is a proper mapping, then $\operatorname{dim} Y \geqq n$. We first observe a more obvious property for $X$.

\section{I.1. Proposition. $\operatorname{dim} \prod_{i=1}^{n} L\left(\omega_{i}\right)=n$.}

Proof. First note that for $1 \leqq i \leqq n, L\left(\omega_{i}\right)$ is sequentially compact. Therefore $\prod_{i=1}^{n} L\left(\omega_{i}\right)$ is countably compact and hence pseudocompact. By the Glicksberg Theorem [4, p. 138], $\beta \prod_{i=1}^{n} L\left(\omega_{i}\right)$ $=\prod_{i=1}^{n} \beta L\left(\omega_{i}\right)$. But $\beta L\left(\omega_{i}\right)$ is just the one point compactification $L\left(\omega_{i}\right)^{*}$ of $L\left(\omega_{i}\right)$ and clearly $\operatorname{dim} L\left(\omega_{i}\right)^{*}=1$. By the product theorem for compact spaces [6, Theorem $26-4$, p. 153], $\operatorname{dim} \prod_{i=1}^{n} \beta L\left(\omega_{i}\right) \leqq n$. Thus $\operatorname{dim} \prod_{i=1}^{n} L\left(\omega_{i}\right) \leqq n$ by $\left[4\right.$, VI.1, p. 98]. Since $\prod_{i=1}^{n} L\left(\omega_{i}\right)$ contains a copy of the $n$-cube $I^{n}, \operatorname{dim} \prod_{i=1}^{n} L\left(\omega_{i}\right)=n$.

To prove the main result of this section we will need the following characterization of dimension.

I.2. Theorem. For a normal space $X, \operatorname{dim} X \leqq n$ if and only if for any $n+1$ disjoint pairs of closed sets $C_{1}, C_{1}^{\prime}, \cdots, C_{n+1}, C_{n+1}^{\prime}$, there exist closed sets $B_{1}, \cdots, B_{n+1}$ such that (i) each $B_{i}$ separates $C_{i}$ and $C_{i}^{\prime}$ and (ii) $\bigcap_{i=1}^{n+1} B_{i}=\varnothing$.

A proof of this theorem can be found in $[6$, p. 51].

I.3. Proposition. Suppose that $X_{i}$ is totally ordered and in the order topology $X_{i}$ is compact and connected for $i=1, \cdots, n$. Let $x_{i}^{+}$and $x_{i}^{-}$ be the maximum and minimum points of $X_{i}$ for each $i$. Let $C_{i}^{\delta}=\left\{\left(x_{j}\right) \in \prod_{j=1}^{n} X_{j}: x_{i}=x_{i}^{\delta}\right\}$ where $\delta=+$ or $\delta=-$. Then if $B_{i}$ is closed and separates $C_{i}^{+}$and $C_{i}^{-}$for $i=1, \cdots, n$, then $\bigcap_{i=1}^{n} B_{i} \neq \varnothing$.

Proof. First observe that there exist maximum and minimum points $x_{i}^{+}$and $x_{i}^{-}$in each $X_{i}$ or there would exist an obvious net having no convergent subnet. Suppose that $B_{i}$ separates $C_{i}^{+}$and $C_{i}^{-}$and that $\bigcap_{i=1}^{n} B_{i}=\varnothing$. Then let $\varrho_{i}=\left\{x_{i}^{0}=x_{i}^{-}<x_{i}^{1}<\cdots<x_{i}^{\boldsymbol{k}_{i}}=x_{i}^{+}\right\} \subset X_{i}$ be chosen for $i=1, \cdots, n$ such that if $F_{i}^{j}=\left\{x \in X_{i}: x_{i}^{j-1} \leqq x \leqq x_{i}^{j}\right\}$ and

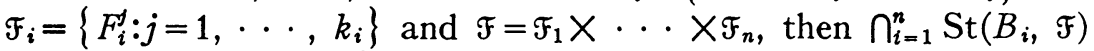
$=\varnothing$ and $\operatorname{St}\left(B_{i}, \mathcal{F}\right) \cap C_{i}^{\delta}=\varnothing$ for $i=1, \cdots, n$ and $\delta=+$ or $\delta=-$ (for notation see $[4$, p. 4$])$. Such a set of $Q_{i}$ 's exist having this property since the stars of covers of the form $\mathcal{F}$ form a basis for the unique uniformity on $\prod_{i=1}^{n} X_{i}$. Now let $I=[0,1]$ and

$$
\mathrm{Q}_{i}^{\prime}=\left\{0=y_{i}^{0}<y_{i}^{1}<\cdots<y_{i}^{k_{i}}=1\right\} \subset I
$$

be any increasing sequence of $k_{i}+1$ elements in $I$. Let 


$$
G_{i}^{j}=\left\{x \in I: x_{i}^{j-1} \leqq x \leqq x_{i}^{j}\right\}
$$

and

$$
\mathcal{F}_{i}^{\prime}=\left\{G_{i}^{j}: j=1, \cdots, k_{i}\right\}
$$

Then let

$$
\mathfrak{F}^{\prime}=\mathfrak{F}_{1}^{\prime} \times \cdots \times \mathfrak{F}_{n}^{\prime}
$$

Let

$$
D_{i}=\bigcup\left\{G_{1}^{j_{1}} \times \cdots \times G_{n}^{j_{n}} \in \mathcal{F}^{\prime}: F_{1}^{j_{1}} \times \cdots \times F_{n}^{j_{n}} \cap B_{i} \neq \varnothing\right\} .
$$

Since $B_{i}$ and thus $\operatorname{St}\left(B_{i}, \mathcal{F}\right)$ separate $C_{i}^{+}$and $C_{i}^{-}$in $\prod_{i=1}^{n} X_{i}$ one can easily show that $D_{i}$ separates $C_{i}^{+}$and $C_{i}^{-}$in $I^{n}=\prod_{j=1}^{n}[0,1]_{j}$. But by [3, IV.1.D, p. 40] we have that $\bigcap_{i=1}^{n} D_{i} \neq \varnothing$. This implies that $\bigcap_{i=1}^{n} \operatorname{St}\left(B_{i}, \mathcal{F}\right)$ is not empty which contradicts the choice of $\mathcal{F}$. Therefore $\bigcap_{i=1}^{n} B_{i} \neq \varnothing$.

We can now prove our main theorem in this section.

I.4. Theorem. Let $X=\prod_{i=1}^{n} L\left(\omega_{i}\right)$ and $f(X)=Y$ be a proper mapping. Then $\operatorname{dim} Y \geqq n$. In fact $Y$ has a compact subset $Y^{\prime}$ with $\operatorname{dim} Y^{\prime} \geqq n$.

Proof. We will first state a claim. We will then show how the theorem follows from the claim. Then we will prove the claim.

Claim. If $X, f$, and $Y$ are as in the theorem, then there are points $z_{i} \in L\left(\omega_{i}\right)$ for $i=1, \cdots, n$ such that if $X_{i}=\left[0, z_{i}\right] \subset L\left(\omega_{i}\right)$ and $C_{i}^{+}=\left\{\left(x_{1}, \cdots, x_{n}\right) \in \prod_{j=1}^{n} X_{j}: x_{i}=z_{i}\right\}$ and $C_{i}^{-}=\left\{\left(x_{1}, \cdots, x_{n}\right)\right.$ $\left.\in \prod_{j=1}^{n} X_{j}: x_{i}=0\right\}$ for $i=1, \cdots, n$, then $f\left(C_{i}^{+}\right) \cap f\left(C_{i}^{-}\right)=\varnothing$ for $i=1, \cdots, n$.

Assuming the claim let $g=f \mid \prod_{i=1}^{n} X_{i}: \prod_{i=1}^{n} X_{i} \rightarrow f\left(\prod_{i=1}^{n} X_{i}\right)=Y^{\prime}$. Then $g$ is also a proper map and has compact domain and range. Suppose that $D_{i}$ is a closed set which separates $f\left(C_{i}^{+}\right)$and $f\left(C_{i}^{-}\right)$in $Y^{\prime}$ for $i=1, \cdots, n$. Then $B_{i}=g^{-1}\left(D_{i}\right)$ separates $C_{i}^{+}$and $C_{i}^{-}$in $\prod_{j=1}^{n} X_{j}$. By Proposition I.3, $\bigcap_{i=1}^{n} B_{i} \neq \varnothing$. Thus $\bigcap_{i=1}^{n} D_{i} \neq \varnothing$. By Theorem I.2, $\operatorname{dim} Y^{\prime} \geqq n$. Thus $\operatorname{dim} Y \geqq n$. We now need only prove the claim and the proof of Theorem I.4 will be complete.

Proof of THE Claim. It will be convenient for purposes of describing the proof to think of $\prod_{i=1}^{0} L\left(\omega_{i}\right)$ as being the point $0 \in L\left(\omega_{1}\right)$. Now consider $\prod_{i=1}^{n} L\left(\omega_{i}\right)$. Note that $\prod_{i=1}^{n-1} L\left(\omega_{i}\right)$ is the union of $\omega_{n-1}$ compact sets $\left\{F_{\alpha}: \alpha<\omega_{n-1}\right\}$. For each $\alpha<\omega_{n-1}$ we define a point $x_{\alpha} \in L\left(\omega_{n}\right)$ as follows. Let $G_{\alpha}=f^{-1} f\left(F_{\alpha} \times\{0\}\right)$. Then $G_{\alpha}$ is a compact subset of $\prod_{i=1}^{n} L\left(\omega_{i}\right)$ since $f$ is a proper map. Let $\pi_{n}: \prod_{i=1}^{n} L\left(\omega_{i}\right)$ 
$\rightarrow L\left(\omega_{n}\right)$ be the projection map. Let $x_{\alpha}=\max \pi_{n}\left(G_{\alpha}\right)$ in $L\left(\omega_{n}\right)$. Now the collection of points $\left\{x_{\alpha}: \alpha<\omega_{n-1}\right\}$ has cardinality at most $\omega_{n-1}$ and thus cardinality strictly less than $\omega_{n}$. Thus the set is bounded above in $L\left(\omega_{n}\right)$. Let $z_{n} \in L\left(\omega_{n}\right)$ be such that $z_{n}>x_{\alpha}$ for all $\alpha<\omega_{n-1}$. Then clearly it must be that

$$
f\left(\prod_{i=1}^{n-1} L\left(\omega_{i}\right) \times\{0\}\right) \cap f\left(\prod_{i=1}^{n-1} L\left(\omega_{i}\right) \times\left\{z_{n}\right\}\right)=\varnothing .
$$

We have now found the $z_{n}$ of the claim. If $n>1$ we continue. Note that $\prod_{i=1}^{n-2} L\left(\omega_{i}\right)$ is the union of $\omega_{n-2}$ compact sets $\left\{F_{\alpha}: \alpha<\omega_{n-1}\right\}$. For each $\alpha<\omega_{n-2}$ we define a point $x_{\alpha} \in L\left(\omega_{n-1}\right)$ as follows. Consider

$$
F_{\alpha} \times\{0\} \times\left[0, z_{n}\right] \subset \prod_{i=1}^{n-2} L\left(\omega_{i}\right) \times L\left(\omega_{n-1}\right) \times L\left(\omega_{n}\right)
$$

Let $G_{\alpha}=f^{-1} f\left(F_{\alpha} \times\{0\} \times\left[0, z_{n}\right]\right)$. Then $G_{\alpha}$ is compact for all $\alpha<\omega_{n-2}$. Let $x_{\alpha}=\max \pi_{n-1}\left(G_{\alpha}\right) \in L\left(\omega_{n-1}\right)$. Then the set $\left\{x_{\alpha}: \alpha<\omega_{n-2}\right\}$ has cardinality at most $\omega_{n-2}$. Thus this set has an upper bound in $L\left(\omega_{n-1}\right)$. Let $z_{n-1}$ be such that $z_{n-1}>x_{\alpha}$ for all $\alpha<\omega_{n-2}$. Then clearly

$f\left(\prod_{i=1}^{n-2} L\left(\omega_{i}\right) \times\{0\} \times\left[0, z_{n}\right]\right) \cap f\left(\prod_{i=1}^{n-2} L\left(\omega_{i}\right) \times\left\{z_{n-1}\right\} \times\left[0, z_{n}\right]\right)=\varnothing$.

If $n>2$ we continue. By repeating this process $n$ times we will finally have $n$ points $z_{i} \in L\left(\omega_{i}\right)$ satisfying the conditions of the claim. This completes the proof of Theorem I.4.

II. The minimum dimension of a compactification. Dowker's Example $M$ described in [1] has given fundamental insight into the relation of the various dimension functions. In [8] and [6, pp. 102$106]$ expanded versions of this example were used to show how ind $X$ and $\operatorname{dim} X$ could vary for normal spaces $X$. The examples we give in this section employ many of the same principles used in Example M together with Theorem I.4. We will try not to belabor the description of our examples since they are very similar to Example M. First note the following lemma.

II.1. Lemma. Let $X=\prod_{i=1}^{n} L\left(\omega_{i}\right)$ and let $X^{*}=X \cup\{\infty\}$ be the one point compactification of $X$. Then $X^{*}=U_{\alpha<\omega_{1}} X_{\alpha}$ where $X_{\alpha} \subset X_{\beta}$ whenever $\alpha<\beta<\omega_{1}$ and ind $X_{\alpha}=0$ for all $\alpha<\omega_{1}$.

Proof. The proof is only sketched. We know that $[0,1)=U_{\alpha<\omega_{1}} Z_{\alpha}$ where the $Z_{\alpha}$ 's have the property that $Z_{\alpha} \subset Z_{\beta}$ for $\alpha<\beta<\omega_{1}$ with $\operatorname{dim} Z_{\alpha}=0$ for all $\alpha<\omega_{1}$. Now consider $L\left(\omega_{i}\right)=\left[0, \omega_{i}\right) \times[0,1)$. Let 
$Y_{i, \alpha}=\left[0, \omega_{i}\right) \times Z_{\alpha}$. Then ind $Y_{i, \alpha}=0$ as a subspace of $L\left(\omega_{i}\right)$. Let $X_{\alpha}^{\prime}=\prod_{i=1}^{n} Y_{i, \alpha}$ and let $X_{\alpha}=X_{\alpha}^{\prime} \cup\{\infty\}$. Then ind $X_{\alpha}=0$ and clearly the $X_{\alpha}$ 's have the other required properties.

It is well known that a space $X$ has a 0 -dimensional compactification if and only if it has ind $X=0$ (see [2,16.D.2, p. 261]). This fact will be needed in the proof of Theorem II.2.

II.2. Theorem. Let $n$ be a positive integer. Then there is a completely regular space $Y$ with $\min \operatorname{dim} Y=n$ and with a point $p \in Y$ with $\min \operatorname{dim} Y-\{p\}=0$.

Proof. The proof is only sketched. Let $X=\prod_{i=1}^{n} L\left(\omega_{i}\right)$ and $X^{*}=X \cup\{\infty\}$ be the one point compactification of $X$. Let $X^{*}=\cup_{\alpha<\omega_{1}} X_{\alpha}$ with ind $X_{\alpha}=0$ and with $X_{\alpha} \subset X_{\beta}$ for $\alpha<\beta<\omega_{1}$. Then let $Y^{\prime}=U_{\alpha<\omega_{1}}\{\alpha\} \times X_{\alpha} \subset\left[0, \omega_{1}\right] \times X^{*}$ and give $Y^{\prime}$ the subspace topology. Clearly ind $Y^{\prime}=0$ and thus $Y^{\prime}$ has a 0 -dimensional compactification. Now if one considers any continuous $f: Y^{\prime} \rightarrow[0,1]$ one can show that $f$ has a continuous extension to $Y^{\prime} \cup\left\{\omega_{1}\right\} \times X^{*}$ $\subset\left[0, \omega_{1}\right] \times X^{*}$ (we leave it to the reader to verify this). Thus if we define $Y=Y^{\prime} \cup\left\{\omega_{1}\right\} \times\{\infty\}$, then $\beta Y=\beta Y^{\prime}$ and in $\beta Y-Y$ there is a closed copy $T$ of $X=\prod_{i=1}^{n} L\left(\omega_{i}\right)$. Now suppose that $Z$ is any compactification for $Y$. Then let $g: \beta Y \rightarrow Z$ be the induced map onto $Z$. Then $g \mid T$ is a proper map. Thus by Theorem I.4 there is a compact set $Q \subset g(T) \subset Z-Y$ such that $\operatorname{dim} Q \geqq n$. Thus $\operatorname{dim} Z \geqq n$. Now $Y$ is the space in the theorem and $p=\left\{\omega_{1}\right\} \times\{\infty\}$ is the point of the theorem. To see that min $\operatorname{dim} Y=n$ simply note that the closure of $Y$ in $\left[0, \omega_{1}\right] \times X^{*}$ is a compactification for $Y$ which has dimension $n$.

II.3. Remark. For $n=\infty$ there is a locally compact space $X$ such that for every proper mapping $f(X)=Y, Y$ is infinite dimensional. Simply let $X$ be the free topological union of $\left\{\prod_{i=1}^{n} L\left(\omega_{i}\right)\right\}_{n=1}^{\infty}$. By a trivial modification $X$ can be made to be connected as well.

We can also easily show that there is a $Y$ with $\min \operatorname{dim} Y=\infty$ with a $p \in Y$ with min $\operatorname{dim} Y-\{p\}=0$. Let $Y_{n}$ be the $Y$ given by Theorem II. 2 for each positive integer $n$ and let $p_{n} \in Y_{n}$ be the point in the theorem. Then let $Y=\left(\cup_{n=1}^{\infty} Y_{n}\right) / R$ where the equivalence relation $R$ identifies all the $p_{n}$ 's to a point $p$ and let $Y$ have the quotient topology. Then $\min \operatorname{dim} Y=\infty$ and $\min \operatorname{dim} Y-\{p\}=0$.

\section{REFERENCES}

1. C. H. Dowker, Local dimension of normal spaces, Quart. J. Math. Oxford Ser. (2) 6 (1955), 101-120. MR 19, 157.

2. L. Gillman and M. Jerison, Rings of continuous functions, The University Series in Higher Math., Van Nostrand, Princeton, N. J., 1960. MR 22 \#6994. 
3. W. Hurewicz and H. Wallman, Dimension theory, Princeton Math. Series, vol. 4, Princeton Univ. Press, Princeton, N. J., 1941. MR 3, 312.

4. J. R. Isbell, Uniform spaces, Math. Surveys, no. 14, Amer. Math. Soc., Providence, R. I., 1964. MR 30 \#561.

5. J. Keesling, Proper mappings and dimension, Proc. Amer. Math. Soc. 29 (1971), 202-204.

6. K. Nagami, Dimension theory, Academic Press, New York, 1970.

7. J. Nagata, Modern dimension theory, Bibliotheca Math., vol. 6, Interscience, New York, 1965. MR 34 \#8380.

8. Ju. M. Smirnov, An example of zero-dimensional normal space having infinite dimensions from the standpoint of covering, Dokl. Akad. Nauk SSSR 123 (1958), 40-42. (Russian) MR 20 \#6087.

University of Florida, Gainesville, Florida 32601 